\title{
HST/FOS DISCOVERY OF PROBABLE CVS IN NGC 6397
}

\author{
JONATHAN E. GRINDLAY \\ Harvard-Smithsonian Center for Astrophysics \\ 60 Garden Street \\ Cambridge, MA 02138, USA
}

AND

ADRIENNE M. COOL

Department of Astronomy

University of California

Berkeley, CA 94720, USA

Our recent HST/FOS spectra (Grindlay et al 1995; GC95) have spectroscopically identified the 3 central $\mathrm{H} \alpha$ candidates (Cool et al 1995; CG95) as the probable cataclysmic variable (CV) counterparts of the the three central dim x-ray sources (Cool et al 1993; CG93) in the nearby core collapsed globular NGC 6397. Here we present the x-ray vs. optical properties of these three objects, derived from the FOS spectra presented in GC95 and the x-ray data of CG93, which strengthen the case that they are CVs and not quiescent LMXBs. Additional data and discussion are presented in the accompanying paper by Grindlay (these proceedings).

CG95 plotted (their Fig. 6) the apparent correlation between the $\mathrm{x}$ ray/optical flux ratio and the equivalent width of the $\mathrm{H} \beta$ emission line. As discussed in Patterson and Raymond (1985; PR), such a correlation is found for CVs locally in the Galaxy. The "standard" correlation between $\mathrm{x}$-ray/optical flux ratio, $\mathrm{f}_{x} / \mathrm{f}_{V}$, and emission line strength uses the equivalent width of $\mathrm{H} \beta$. Our WFPC1 images provided only $\mathrm{H} \alpha$ and $\mathrm{R}$ band magnitudes so that $\mathrm{f}_{V}$ was estimated from assuming $(\mathrm{V}-\mathrm{R})=0.5$, while $\mathrm{EW}(\mathrm{H} \beta)$ was assumed equal to $\mathrm{EW}(\mathrm{H} \alpha)$. With our FOS spectra now actually covering both $\mathrm{H} \beta$ and the $\mathrm{V}$ band continuum (cf. GC95), the ratio can be measured (cf. Fig. 1). Compared with the WFPC1-derived points of CG95 (plotted here as open symbols), the FOS points lie significantly closer to the correlation line (solid) of PR and in fact within the factor of 3 variation region (dashed). 
The x-ray/optical flux ratio vs. $\mathrm{EW}(\mathrm{H} \beta)$ plot may help to further discriminate quiescent LMXBs from CVs for the cluster dim sources (cf. GC95). For CVs, as the accretion rate increases onto the WD, the innermost accretion disk or boundary layer must become increasingly optically thick so that the continuum radiation is both increased and the Balmer lines eventually go into absorption. Conversely, at low accretion rates, the disk surface or corona contributes more of the visible flux and the relatively optically thin higher temperature gas ensures the lines are in emission. Thus in quiescent LMXBs, with their very much lower accretion rate (for dim sources), the lines are expected to be relatively strong and the optical continuum weak. Indeed, taking the ROSAT detection of the quiescent LMXB Cen X-4 (Verbunt et al 1994) as corresponding to the optical continuum and $\mathrm{H} \beta$ flux values of McClintock and Remillard (1990), the point is well above the CV correlation line in Figure 1.

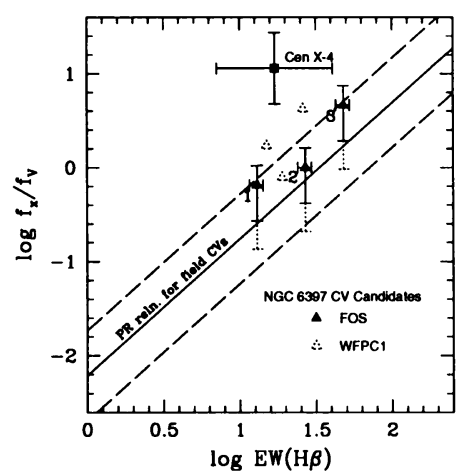

Figure 1. $\mathrm{H} \beta$ emission strength (equivalent width) vs. $\mathrm{x}$-ray/optical flux for $3 \mathrm{CV}$ candidates in NGC 6397 vs. field CVs and the quiescent LMXB Cen X-4.

The FOS spectra therefore support the identification of the cluster dim sources with CVs. The presence of moderately strong He I and He II lines, with $\mathrm{EW}(\mathrm{He} \mathrm{II}) \sim 0.3-0.5 \times \mathrm{EW}(\mathrm{H} \beta)$ are very similar to the values found by Silber (1992) for magnetic (DQ Her type) CVs as discussed in more detail in the accompanying paper by Grindlay.

This work was supported by grants HST GO-5497 and NASA NAGW-3280.

\section{References}

Cool, A. et al 1993, ApJ, 410, L103 (CG93).

Cool, A. et al 1995, ApJ, 439, 695 (CG95).

Grindlay, J., Cool, A. et al 1995, ApJ, 455, L47 (GC95).

McClintock, J. and Remillard, R. 1990, ApJ, 350, 386.

Patterson, J. and Raymond, J. 1985, ApJ, 292, 535 (PR).

Silber, A. 1992, Ph.D. Thesis, MIT.

Verbunt, F. et al 1994, A\&A, 285, 903. 